\title{
COUNCIL AND BOARD OF TRUSTEES. 1975 and 1976
}

Except for the Members-at-Large of the Council, the month and year of the first term and the end of the present term are given. For Members-at-Large of the Council, the last year of the present term is listed.

\section{COUNCIL}

President: Lipman Bers 1/75-12/76

Past President: Saunders Mac Lane 1/75-12/75 President-elect: R. H. Bing 1/76-12/76

Vice Presidents:

Raoul H. Bott 1/74-12/75

Mary W. Gray 1/76-12/77

Irving Kaplansky 1/74-12/75

John W. Milnor 1/75-12/76

Louis Nirenberg 1/76-12/77
Secretary: Everett Pitcher 1/67-12/76

Associate Secretaries:

Paul Bateman 1/67-12/77

Walter Gottschalk 1/71-12/76

Orville G. Harrold, Jr. 1/65-12/76

Kenneth A. Ross 1/71-12/77

Treasurer: Franklin Pererson 1/73-12/76

Associate Treasurer:

Murray H. Protter 1/73-12/76

Publications and Communications Committees

\section{Bulletin Editorial Committee}

Paul R. Halmos 1/74-12/76

John L. Kelley 1/73-12/75

Olga Taussky 1/76-12/78

Hans F. Weinberger 1/72-12/77

Proceedings Editorial Committee

Glen E. Bredon 1/72-12/75

Thomas A. Chapman 1/76-12/79

W. Wistar Comfort 1/72-12/75

Chandler Davis 9/74-12/76

Robert M. Fossum 1/74-12/77

Richard R. Goldberg 1/73-12/79

Richard K. Miller 9/74-12/78

Barbara L. Osofsky 1/74-12/77

Joseph A. Wolf 1/76-12/79

Transactions and Memoirs Editorial Committee

Alexandra Bellow 10/74-12/77

Philip T. Church 1/74-12/77

Solomon Feferman 1/76-12/79

Alistair H. Lachlan 1/72-12/75

Robert T. Seeley 1/76-12/79

Stephen S. Shatz 1/75-12/78

Daniel W. Stroock 1/75-12/78

François Treves $1 / 72-12 / 75$
Representatives on American Journal of Mathematics

Hyman Bass 1/71-12/76

I. M. Singer 1/72-12/77

Mathematical Reviews Editorial Committee Robert G. Bartle 1/74-12/76

D. J. Lewis 1/75-12/77

Jacob T. Schwartz 1/73-12/78

Mathematical Surveys Editorial Committee

Robert G. Bartle 1/72-12/77

Edgar H. Brown, Jr. 1/68-12/76

Paul R. Halmos 1/73-12/75

Maxwell A. Rosenlicht 1/76-12/78

Mathematics of Computation Editorial

Committee

James H. Bramble 1/73-12/77

Walter Gautschi 1/75-12/77

Alston S. Householder 1/73-12/76

John W. Wrench, Jr. 1/73-12/78

Colloquium Editorial Committee

Alberto P. Calderón 1/71-12/76

S. S. Chern $1 / 72-12 / 77$

Samuel Eilenberg 1/73-12/78

Chairman, Committee to Monitor Problems in Communication Leonard Gillman 1/75-12/77 


\section{Members-at-Large}

All terms are for 3 years and expire on December 31 of the given year.

1975

Anatole Beck

Michael Golomb

Mary W. Gray

Arthur P. Mattuck

Cathleen S. Morawetz

1977

David Gale

Judy Green

Phillip A. Griffiths

Karl K. Norton

J. Ernest Wilkins, Jr.
1976

Charles W. Curtis

Herbert B. Keller

Robion C. Kirby

Lee Lorch

Jane Cronin Scanlon

1978

Hugo Rossi

Lee A. Rubel

Barry Simon

Daniel Waterman

Guido L. Weiss

\section{BOARD OF TRUSTEES}

Paul T. Bateman 1/71-12/75

Lipman Bers (ex officio) 1/75-12/76

Calvin C. Moore 1/71-12/79

Cathleen S. Morawetz 1/76-12/80
Richard S. Palais 1/72-12/76

Franklin P. Peterson (ex officio) 8/73-12/76

Murray H. Protter (ex officio) 1/73-12/76

Alex Rosenberg 1/74-12/78

Abraham H. Taub 1/73-12/77 
